\title{
Prion Diseases in Animals and Zoonotic Potential
}

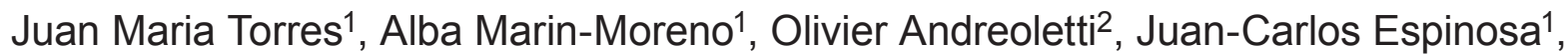 \\ Vincent Beringue $^{3}$, Patricia Aguilar ${ }^{1}$, and Natalia Fernandez-Borges ${ }^{1}$ \\ ${ }^{1}$ Centro de Investigacion en Sanidad Animal (CISA-INIA), Valdeolmos, Madrid, Spain \\ 2UMR INRA-ENVT 1225 Interactions Hotes Agents Pathògenes, ENVT; Toulouse, France \\ 3UR892, Virologie Immunologie Moléculaires; Jouy-en-Josas, France
}

Dietary exposure to Bovine Spongiform Encephalopathy (BSE) contaminated bovine tissues is considered as the origin of variant Creutzfeldt-Jakob disease (vCJD) in humans. To date, BSE agent is the only recognized zoonotic prion. Despite the variety of transmissible spongiform encephalopathy (TSE) agents that have been circulating for centuries in farmed ruminants, there is no apparent epidemiological link between exposure to ruminant products and the occurrence of other form of TSE in human like sporadic Creutzfeldt-Jakob disease (sCJD). However, the zoonotic potential of the diversity of circulating TSE agents has never been systematically assessed. The major issue in experimental assessment of TSEs zoonotic potential lies in the modelling of the "species barrier," the biological phenomenon that limits TSE agents' propagation from one species to another. In the past decade, mice genetically engineered to express normal forms of the human prion protein have proven to be essential in studying human prions pathogenesis and modelling the capacity of TSEs to cross the human species barrier.

To assess the zoonotic potential of prions circulating in farmed ruminants, we study their transmission ability in transgenic mice expressing human $\operatorname{PrP}^{\mathrm{C}}$ (HuPrP-Tg). Two lines of mice expressing different forms of the human $\mathrm{PrP}^{\mathrm{C}}(129 \mathrm{Met}$ or $129 \mathrm{Val})$ are used to determine the role of the Met129Val dimorphism in susceptibility/resistance to the different agents.

These transmission experiments confirm the ability of BSE prions to propagate in 129M-HuPrP-Tg mice and demonstrate that Met129 homozygotes may be susceptible to BSE in sheep or goats to a greater degree than the BSE agent in cattle, and that these agents can convey molecular properties and be neuropathologically indistinguishable from vCJD. However, homozygous $129 \mathrm{~V}$ mice are resistant to all tested BSE derived prions independently of the originating species, suggesting a higher transmission barrier for 129V-PrP variant.

Transmission data also revealed that several scrapie prions propagate in HuPrP-Tg mice with efficiency comparable to that of cattle BSE. While the efficiency of transmission at primary passage was low, subsequent passages resulted in a highly virulent prion disease in both Met129 and Val129 mice. Transmission of the different scrapie isolates in these

Received: 8 July 2016; Accepted: 5 October 2016; Published online: 7 December 2016

Corresponding author: Juan Maria Torres, Centro de Investigacion en Sanidad Animal (CISA-INIA), Valdeolmos, Madrid, Spain (jmtorres@inia.es)

The contents of this article reflect solely the view of the author(s).

Conflict of interest statement: The authors had no conflicts of interest to declare in this article.

This paper was presented at the Animal Prion Diseases Workshop "Updated Diagnosis and Epidemiology of Animal Prion Diseases for Food Safety and Security" supported by the OECD Co-operative Research Programme.

Abbreviations: BSE: Bovine Spongiform Encephalopathy; PrPc: cellular protein; PrPsc: infectious protein; sCJD: sporadic Creutzfeldt-Jakob disease; TSE: transmissible spongiform encephalopathy; vCJD: variant Creutzfeldt-Jakob disease 
mice leads to the emergence of prion strain phenotypes that showed similar characteristics to those displayed by MM1 or VV2 sCJD prion. These results demonstrate that scrapie prions have a zoonotic potential and raise new questions about the possible link between animal and human prions.

Key words: BSE, PrP, sCJD, TSE, vCJD

\section{Introduction}

Transmissible spongiform encephalopathies (TSE) encompass a group of neurodegenerative prion diseases affecting different animal species, including humans. They are all characterized by the accumulation in the brain of an infectious protein $\left(\mathrm{PrP}^{\mathrm{Sc}}\right)$, highly insoluble, corresponding to the pathogenic isoform of the cellular protein $\left(\operatorname{PrP}^{\mathrm{C}}\right)$ and whose function is not well understood ${ }^{1-3}$. Within the group of TSE, scrapie in sheep and goats occupies a prominent position. This TSE has been known for more than two centuries, and is more widespread in EU countries ${ }^{4,5)}$. Several experimental transmission studies in mice have demonstrated the existence of significant biodiversity between different scrapie agents $^{6}$. Furthermore, in 1998, a new type of scrapie was diagnosed in sheep in Norway that was later called scrapie Nor9 $8^{7)}$ and now also known as atypical scrapie (type-Nor98). Although most cases have been detected in sheep, atypical scrapie cases have also been reported in goats ${ }^{5)}$. Since the implementation in Europe of a broad screening program for TSEs in small ruminants, atypical scrapie has been identified throughout the European Union ${ }^{8-14)}$, which detected a significant number of cases of atypical scrapie in small ruminants, even exceeding that of classical scrapie cases.

The agent of bovine spongiform encephalopathy (BSE) is an epidemic prion that has killed more than 200,000 cattle, mainly in the UK. Transmission of BSE to humans through contaminated food was responsible for the variant of Creutzfeldt-Jakob disease (vCJD) ${ }^{15,16)}$ triggering a major Food Safety crisis. Recently, two atypical forms of BSE have been identified in several European countries ${ }^{17)}$, Japan ${ }^{18,19)}$, the United States ${ }^{20)}$, and Canada ${ }^{21)}$. Several studies show that these atypical forms are associated with two strains of different prions that are mainly characterized by their PrPres that have different electrophoretic profiles, calling them H-type and L-type according to the electrophoretic migration of their unglycosylated $\mathrm{PrP}^{\mathrm{res}}$ compared to the conventional type (C-type). All the epidemiological and biological evidence suggests that H-BSE and L-BSE represent sporadic forms of BSE associated with two strains of different prions ${ }^{22-24)}$.

To date, the agent responsible for BSE is the only recognized zoonotic prion, having led to the emergence of vCJD. Despite the variety of TSE agents circulating for centuries in small ruminant farms, any apparent epidemiological link has been found between the consumption of ruminants and the occurrence of other TSEs forms in humans. However, the zoonotic potential of the diversity of the current TSE agents has never been evaluated with the necessary rigor. Furthermore, the etiology of sporadic TSE in humans is unknown.

The most important thing in assessing the zoonotic potential of TSEs aspect is the development of appropriate models that mimic the species barrier (the biological phenomenon that limits the spread of TSE from one species to another). Differences in amino-acid sequence between host $\mathrm{PrP}^{\mathrm{C}}$ and donor $\mathrm{PrP}^{\mathrm{Sc}}$ are the main drivers for the transmission barrier. However, it is also well established that strain properties have a determinant impact on the ability of prions to cross the species barrier.

Conceptually, the permeability of the prion transmission barrier is considered to be driven at the molecular level by the conformational compatibility between host $\mathrm{PrP}^{\mathrm{C}}$ and the misfolded strain-specific protein assemblies of $\operatorname{PrP} \mathrm{Pc}^{\mathrm{Sc}}$ that are present within infectious prion particle ${ }^{25}$. As a consequence, mice genetically engineered to express human $\operatorname{PrP}$ (HuPrP-Tg), in the absence of endogenous mouse PrP, have emerged as relevant animal models to assess prion transmission across the human species barrier ${ }^{26)}$. A major determinant of the susceptibility to human prion disease is Met/Val dimorphism at codon 129 of the PRNP gene ${ }^{27,28)}$, and HuPrP-Tg mice lines exist that express these variants of human $\mathrm{PrP}^{29}$. These HuPrP-Tg mouse lines propagate human prions without an apparent species barrier and have been successfully used to confirm the zoonotic ability of the BSE agent from cattle ${ }^{16,30,31)}$.

We have developed three different HuPrP-Tg mouse models: i) HuPrP-Tg340-Met ${ }_{129}\left(\operatorname{TgMet}_{129}\right)$ transgenic mouse line expressing human $\mathrm{PrP}^{\mathrm{C}}$ at a 4 -fold level ${ }^{30)}$ ii) HuPrP-Tg361-Val ${ }_{129}\left(\mathrm{TgVal}_{129}\right)$ transgenic mouse line expressing human $\mathrm{PrP}^{\mathrm{C}}$ at a 4 -fold level ${ }^{32}$, and iii) HuPrP-Tg351-Met/Val ${ }_{129}\left(\mathrm{TgMet} / \mathrm{Val}_{129}\right)$ transgenic mouse obtained by mating $\mathrm{TgMet}_{129}$ and $\mathrm{TgVal}_{129}$ mice; all of them express the same level of transgenic PrP on a mouse PrP null background. These mouse models are being used to assess the zoonotic potential of the diversity of animal prion strains from different farmed species. From each species, a panel of isolates has been selected on the basis of their phenotype diversity trying to include as much diversity as possible. The results allow to determine human susceptibility to these TSE agents (us- 
ing classical BSE as a reference) and to pinpoint any potential etiological link between human prion diseases and TSEs agents from farmed ruminants.

\section{Classical BSE in HuPrP-Tg Mice}

BSE can be transmitted to human PrP TgMet mice although with a high transmission barrier ${ }^{30}$. The transmission efficiency of cattle BSE isolates in this mouse model was low. With all tested BSE isolates, very low attack rates were obtained on primary transmission to these mice. Three passages were necessary to achieve a degree of fitness comparable to VCJD in the same mouse line. This low BSE transmission efficiency to human PrP transgenic mice has also been described by others ${ }^{31,33,34)}$, and suggests a strong although not absolute transmission barrier. Transmission of cattle BSE to another model of human $\mathrm{PrP}$ mice with a more pronounced expression of $\mathrm{PrP}^{\mathrm{C}}$ in the lymphoid tissue has shown that BSE transmission barrier to human might be less pronounced in the spleen tissue than in the brain tissue ${ }^{35}$ and might explain the high prevalence of asymptomatic individuals accumulating prions in their lymphoid organs $\mathrm{s}^{36}$.

Remarkably, a different picture emerged when the sheep and goat BSE isolates were inoculated to human PrP transgenic mouse models ${ }^{30)}$. Attack rates approaching $100 \%$ were observed from the primary passage onwards and mean incubation times were more consistent with those measured after transmission of vCJD. On further passaging, the neuropathological phenotype and $\mathrm{PrP}^{\mathrm{Sc}}$ type of cattle and sheep/goat BSE agents appeared indistinguishable from the vCJD agent propagated in these mice, as previously demonstrated in bovine transgenic mice ${ }^{37)}$, thus strongly supporting the view that the same BSE prion strain has been propagated whatever the infecting species.

These results suggest that the possibility of a small ruminant BSE prion as VCJD causal agent could not be ruled out, and that the risk for humans of a potential goat and/or sheep BSE agent should not be underestimated.

By contrast, although some experiments are still ongoing, TgVal129 mice seem to be resistant to all tested BSE derived prions independently of the originating species. Thus, suggesting a higher transmission barrier for 129Val-PrP variant than for 129Met-PrP variant. This Val129 transmission barrier seems not to be enough to protect heterozygous Met/Val129 individuals from BSE prions infection as some isolates can infect these heterozygous mice although with an evident lower efficiency than homozygous TgMet mice. However, BSE as vCJD was able to be transmitted in TgVal and TgMet/Val after adaptation/amplification in TgMet; thus suggesting that secondary transmission of vCJD can occur in both heterozygous 129Met/Val and homozygous 129Val/Val humans.

Although the exact characteristics and further evolution of the VCJD epidemic still entail uncertainties owing to prolonged incubation times, this apparent high transmission barrier of humans to cattle BSE might be an explanation for the currently low vCJD incidence, considering the high exposure to BSE during the "mad cow" crisis.

\section{Atypical BSE in HuPrP-Tg Mice}

The results obtained in our laboratory up to now show that H-type atypical BSE is unable to infect HuPrP-Tg mice. Other authors also showed that H-BSE isolates failed to infect another line of "humanised" mice (Met129 PrP) $)^{38)}$. In both cases, these mice over-express human PrP and were inoculated intracerebrally with a low dilution inoculum, supporting the view that the transmission barrier of H-BSE from cattle to humans might be quite robust.

Our results show that L-Type atypical BSE is efficiently transmitted to human PrP TgMet with lower transmission barrier than classical-BSE. Other authors also showed that L-BSE field isolates produced TSE disease in two lines of mice over-expressing human $\operatorname{PrP}$ (Met129), exhibiting a molecular phenotype distinct from that of classical BSE ${ }^{33,38)}$. In one of them, as in our results, the L-BSE agent appeared to propagate with no obvious transmission barrier: a 100\% attack rate was observed on first passage, the incubation time was not reduced on subsequent passaging ${ }^{38)}$ and the L-BSE $\mathrm{PrP}^{\mathrm{Sc}}$ biochemical signature was essentially conserved, appearing to be indistinguishable from that seen after experimental inoculation of MM2 sporadic Creutzfeldt-Jakob disease (sCJD) in these mice ${ }^{39}$.

By contrast, L-BSE agent was unable to infect neither TgVal nor TgMet/Val in any of the experiments carried out up to now in our laboratory. Thus, suggesting that Val129 PrP variant could have a protective effect against this agent. 


\section{Classical Scrapie in HuPrP-Tg Mice}

Transmission experiments also revealed that some scrapie isolates can be transmitted to TgMet mice with a transmission barrier comparable to that of BSE in these mice ${ }^{32}$. However, the polymorphic variant Val129 (TgVal) was unable to be infected in primary passage with any of the scrapie isolates tested in this study. Thus, suggesting a higher transmission barrier for Val129 than for Met129 variant. However, this Val129 barrier was not enough to protect heterozygous Met/Val129 individuals. Secondary transmissions from TgMet mice can occur in both TgMet129 and Val129 without apparent transmission barrier. Transmission of these scrapie isolates in HuPrP-Tg mice leads to the emergence of prion strain phenotypes that showed similar characteristics to those displayed by MM1 or VV2 sCJD prion isolates passaged in the same mouse lines ${ }^{32}$.

Do these transmission results in HuPrP-Tg mice imply that sheep scrapie is the cause of SCJD cases in humans? This question challenges the well-established dogma that SCJD is a spontaneous disorder unrelated to animal prion disease. In our opinion, these data on their own do not unequivocally establish a causative link between natural exposure to sheep scrapie and the subsequent appearance of sCJD in humans. However, our studies clearly point out the need to reconsider this possibility. Consequently, it is our opinion that even if a causative link was established between sheep scrapie exposure and the occurrence of certain SCJD cases, it would be wrong to consider small ruminant TSE agents as a new major threat for public health. Despite this, it remains clear that our data provide a new impetus to establish the true zoonotic potential of animal prions.

\section{Acknowledgements}

This work was funded by the EU FP7 project "Priority CT2009-222887," the UK Food Standards Agency grant M03043, the Spanish Plan Nacional de I+D+I project (RTA2012-00004), and the Alliance Biosecure Foundation grant FABS201403.

\section{References}

1. Prusiner SB. Prions. Proc Natl Acad Sci U S A. 1998; 95: 13363-13383. [Medline]

2. Caughey BW, Dong A, Bhat KS, Ernst D, Hayes SF, Caughey WS. Secondary structure analysis of the scrapie-associated protein PrP 27-30 in water by infrared spectroscopy. Biochemistry. 1991; 30: 7672-7680. [Medline]

3. Pan KM, Baldwin M, Nguyen J, et al. Conversion of alpha-helices into beta-sheets features in the formation of the scrapie prion proteins. Proc Natl Acad Sci U S A. 1993; 90: 10962-10966. [Medline]

4. Fediaevsky A, Tongue SC, Nöremark M, Calavas D, Ru G, Hopp P. A descriptive study of the prevalence of atypical and classical scrapie in sheep in 20 European countries. BMC Vet Res. 2008; 4: 19. [Medline]

5. Vaccari G, Panagiotidis $\mathrm{CH}$, Acin C, et al. State-of-the-art review of goat TSE in the European Union, with special emphasis on PRNP genetics and epidemiology. Vet Res. 2009; 40: 48. [Medline]

6. Bruce ME. Scrapie strain variation and mutation. Br Med Bull. 1993; 49: 822-838. [Medline]

7. Benestad SL, Sarradin P, Thu B, Schönheit J, Tranulis MA, Bratberg B. Cases of scrapie with unusual features in Norway and designation of a new type, Nor98. Vet Rec. 2003; 153: 202-208. [Medline]

8. Buschmann A, Biacabe AG, Ziegler U, et al. Atypical scrapie cases in Germany and France are identified by discrepant reaction patterns in BSE rapid tests. J Virol Methods. 2004; 117: 27-36. [Medline]

9. De Bosschere H, Roels S, Benestad SL, Vanopdenbosch E. Scrapie case similar to Nor98 diagnosed in Belgium via active surveillance. Vet Rec. 2004; 155: 707-708. [Medline]

10. Gavier-Widén D, Nöremark M, Benestad S, et al. Recognition of the Nor98 variant of scrapie in the Swedish sheep population. J Vet Diagn Invest. 2004; 16: 562-567. [Medline]

11. Onnasch H, Gunn HM, Bradshaw BJ, Benestad SL, Bassett HF. Two Irish cases of scrapie resembling Nor98. Vet Rec. 2004; 155: 636-637. [Medline]

12. Orge L, Galo A, Machado C, et al. Identification of putative atypical scrapie in sheep in Portugal. J Gen Virol. 2004; 85: 3487-3491. [Medline]

13. Benestad SL, Arsac JN, Goldmann W, Nöremark M. Atypical/Nor98 scrapie: properties of the agent, genetics, and epidemiology. Vet Res. 2008; 39: 19. [Medline]

14. Vidal E, Tortosa R, Costa C, et al. Lack of $\operatorname{Pr}^{\mathrm{Sc}}$ immunostaining in intracranial ectopic lymphoid follicles in a sheep with concomitant non-suppurative encephalitis and Nor98-like atypical scrapie: a case report. Vet J. 2008; 177: 283-288. [Medline]

15. Prusiner SB. Prion diseases and the BSE crisis. Science. 1997; 278: 245-251. [Medline] 
16. Hill AF, Desbruslais M, Joiner S, et al. The same prion strain causes vCJD and BSE. Nature. 1997; 389: 448-450, 526. [Medline]

17. Jacobs JG, Langeveld JP, Biacabe AG, et al. Molecular discrimination of atypical bovine spongiform encephalopathy strains from a geographical region spanning a wide area in Europe. J Clin Microbiol. 2007; 45: 1821-1829. [Medline]

18. Yamakawa Y, Hagiwara K, Nohtomi K, et al. Expert Committee for BSE Diagnosis, Ministry of Health, Labour and Welfare of Japan Atypical proteinase K-resistant prion protein $\left(\mathrm{PrP}^{\mathrm{res}}\right)$ observed in an apparently healthy 23-month-old Holstein steer. Jpn J Infect Dis. 2003; 56: 221-222. [Medline]

19. Masujin K, Shu Y, Yamakawa Y, et al. Biological and biochemical characterization of L-type-like bovine spongiform encephalopathy (BSE) detected in Japanese black beef cattle. Prion. 2008; 2: 123-128. [Medline]

20. Richt JA, Kunkle RA, Alt D, et al. Identification and characterization of two bovine spongiform encephalopathy cases diagnosed in the United States. J Vet Diagn Invest. 2007; 19: 142-154. [Medline]

21. Dudas S, Yang J, Graham C, et al. Molecular, biochemical and genetic characteristics of BSE in Canada. PLoS One. 2010; 5: e10638. [Medline]

22. Béringue $\mathrm{V}$, Bencsik A, Le Dur A, et al. Isolation from cattle of a prion strain distinct from that causing bovine spongiform encephalopathy. PLoS Pathog. 2006; 2: e112. [Medline]

23. Buschmann A, Gretzschel A, Biacabe AG, et al. Atypical BSE in Germany - proof of transmissibility and biochemical characterization. Vet Microbiol. 2006; 117: 103-116. [Medline]

24. Capobianco R, Casalone C, Suardi S, et al. Conversion of the BASE prion strain into the BSE strain: the origin of BSE? PLoS Pathog. 2007; 3: e31. [Medline]

25. Collinge J, Clarke AR. A general model of prion strains and their pathogenicity. Science. 2007; 318: 930-936. [Medline]

26. (BIOHAZ), EFSAoBH, Joint scientific opinion on any possible epidemiological or molecular association between TSEs in animals and humans. EFSA Journal. 2011; 9: 1945-2056. [cited 11 Sep 2013].

27. Windl O, Dempster M, Estibeiro JP, et al. Genetic basis of Creutzfeldt-Jakob disease in the United Kingdom: a systematic analysis of predisposing mutations and allelic variation in the PRNP gene. Hum Genet. 1996; 98: 259-264. [Medline]

28. Mead S, Poulter M, Uphill J, et al. Genetic risk factors for variant Creutzfeldt-Jakob disease: a genome-wide association study. The Lancet Neurology. 2009; 8: 57-66. [Medline]

29. Groschup MH, Buschmann A. Rodent models for prion diseases. Vet Res. 2008; 39: 32. [Medline]

30. Padilla D, Béringue V, Espinosa JC, et al. Sheep and goat BSE propagate more efficiently than cattle BSE in human PrP transgenic mice. PLoS Pathog. 2011; 7: e1001319. [Medline]

31. Asante EA, Linehan JM, Desbruslais M, et al. BSE prions propagate as either variant CJD-like or sporadic CJD-like prion strains in transgenic mice expressing human prion protein. EMBO J. 2002; 21: 6358-6366. [Medline]

32. Cassard H, Torres JM, Lacroux C, et al. Evidence for zoonotic potential of ovine scrapie prions. Nat Commun. 2014 ; 5: 5821. [Medline]

33. Kong Q, Zheng M, Casalone C, et al. Evaluation of the human transmission risk of an atypical bovine spongiform encephalopathy prion strain. J Virol. 2008; 82: 3697-3701. [Medline]

34. Bishop MT, Hart P, Aitchison L, et al. Predicting susceptibility and incubation time of human-to-human transmission of vCJD. Lancet Neurol. 2006; 5: 393-398. [Medline]

35. Béringue V, Herzog L, Jaumain E, et al. Facilitated cross-species transmission of prions in extraneural tissue. Science. 2012; 335: 472-475. [Medline]

36. Gill ON, Spencer Y, Richard-Loendt A, et al. Prevalent abnormal prion protein in human appendixes after bovine spongiform encephalopathy epizootic: large scale survey. BMJ. 2013; 347: f5675. [Medline]

37. Espinosa JC, Andréoletti O, Castilla J, et al. Sheep-passaged bovine spongiform encephalopathy agent exhibits altered pathobiological properties in bovine-PrP transgenic mice. J Virol. 2007; 81: 835-843. [Medline]

38. Béringue V, Herzog L, Reine F, et al. Transmission of atypical bovine prions to mice transgenic for human prion protein. Emerg Infect Dis. 2008; 14: 1898-1901. [Medline]

39. Casalone C, Zanusso G, Acutis P, et al. Identification of a second bovine amyloidotic spongiform encephalopathy: molecular similarities with sporadic Creutzfeldt-Jakob disease. Proc Natl Acad Sci U S A. 2004; 101: 3065-3070. [Medline] 